\title{
On the Turán density of $\{1,3\}$-Hypergraphs
}

\author{
Shuliang Bai \\ Center of Mathematical Sciences and Applications \\ Harvard University \\ Cambridge, U.S.A \\ sbai525@cmsa.fas.harvard.edu
}

\author{
Linyuan $\mathrm{Lu}^{*}$
}

Department of Mathematics

University of South Carolina

South Carolina, U.S.A

lu@math.sc.edu

Submitted: Aug 8, 2018; Accepted: Feb 4, 2019; Published: Mar 8, 2019

(c) The authors.

\begin{abstract}
In this paper, we consider the Turán problems on $\{1,3\}$-hypergraphs. We prove that a $\{1,3\}$-hypergraph is degenerate if and only if it's $H_{5}^{\{1,3\}}$-colorable, where $H_{5}^{\{1,3\}}$ is a hypergraph with vertex set $V=[5]$ and edge set $E=\{\{2\},\{3\},\{1,2,4\}$, $\{1,3,5\},\{1,4,5\}\}$. Using this result, we further prove that for any finite set $R$ of distinct positive integers, except the case $R=\{1,2\}$, there always exist non-trivial degenerate $R$-graphs. We also compute the Turán densities of some small $\{1,3\}$ hypergraphs.
\end{abstract}

Mathematics Subject Classifications: 5D05, 05C65, 05D40

\section{Background}

Turán theory is an important and active area in the extremal combinatorics. In 1941, Turán [10] determined the graph with maximum number of edges among all simple graphs on $n$ vertices that doesn't contain the complete graph $K_{\ell}$ as a sub-graph. The Turán density $\pi(H)$ of a graph $H$ is the least number $\alpha$ so that any large graph with edge density $(\alpha+\epsilon)$ will always contain a sub-graph isomorphic to $H$, for any $\epsilon>0$. Erdős-SimonovitsStone theorem [1,2] determined the Turán densities of all non-bipartite graphs. (The Turán density of any bipartite graph is always 0 . Those are called the degenerate graphs.)

Turán problems on uniform hypergraphs have been actively studied for many decades. However, on non-uniform hypergraphs, these problems are rarely considered. Johnston and $\mathrm{Lu} \mathrm{[4]} \mathrm{established} \mathrm{the} \mathrm{framework} \mathrm{of} \mathrm{the} \mathrm{Turán} \mathrm{theory} \mathrm{for} \mathrm{non-uniform} \mathrm{hypergraphs.}$ A hypergraph $H=(V, E)$ consists of a vertex set $V$ and an edge set $E \subseteq 2^{V}$. Here the edges of $E$ could have different cardinalities. The set of all the cardinalities of edges in

*This author was supported in part by NSF grant DMS 1600811. 
$H$ is denoted by $R(H)$, the set of edge types. In this paper, we will fix a finite set $R$ of positive integers and consider all simple hypergraphs $H$ with $R(H) \subseteq R$, which are called $R$-hypergraphs (or $R$-graphs, for short). We say a hypergraph is simple if there is at most one edge connecting any collection of vertices. A general hypergraph allows every edge to be a multi-set of vertices.

For example, $\{2\}$-graphs are just graphs and $\{r\}$-graphs are just $r$-uniform hypergraphs. An $R$-graph $H$ on $n$ vertices is denoted as $H_{n}^{R}$. We denote $H^{r}$ as the $r$ th level hypergraph of $H$ which consists of all edges of cardinality $r$ of $H$. We denote $K_{n}^{R}$ as the

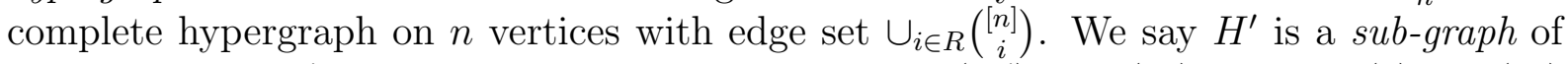
$H$, denoted by $H^{\prime} \subseteq H$, if there exists a 1-1 map $f: V\left(H^{\prime}\right) \rightarrow V(H)$ so that $f(e) \in E(H)$ for any $e \in E\left(H^{\prime}\right)$. A necessary condition for $H^{\prime} \subseteq H$ is $R\left(H^{\prime}\right) \subseteq R(H)$. A chain $C^{R}$ is a special $R$-graph containing exactly one edge of each size such that any pair of these edges are comparable under inclusion relation.

To measure the edge density of a non-uniform hypergraph, we use the Lubell function, which is the expected number of edges in the hypergraph hit by a random full chain [4]. For a non-uniform hypergraph $G$ on $n$ vertices, the Lubell function of $G$ is defined by

$$
h_{n}(G):=\sum_{e \in E(G)} \frac{1}{\left(\begin{array}{c}
n \\
|e|
\end{array}\right)}=\sum_{r \in R(G)} \frac{\left|E\left(G^{r}\right)\right|}{\left(\begin{array}{l}
n \\
r
\end{array}\right)} .
$$

Given a family of hypergraphs $\mathcal{H}$ with common set of edge types $R$, we say $G$ is $\mathcal{H}$-free if $G$ doesn't contain any member of $\mathcal{H}$ as a sub-graph. Let $\pi_{n}(\mathcal{H})$ be the maximum edge density of any $\mathcal{H}$-free $R$-graph on $n$ vertices. The Turán density of $\mathcal{H}$ is defined to be:

$$
\begin{aligned}
\pi(\mathcal{H}) & =\lim _{n \rightarrow \infty} \pi_{n}(\mathcal{H}) \\
& =\lim _{n \rightarrow \infty} \max \left\{h_{n}(G):|v(G)|=n, G \subseteq K_{n}^{R}, \text { and } G \text { is } \mathcal{H} \text {-free }\right\}
\end{aligned}
$$

A hypergraph $G:=G_{n}^{R}$ is extremal with respect to the family $\mathcal{H}$ if $G$ is $\mathcal{H}$-free and $h_{n}(G)$ is maximized.

$\mathrm{Lu}$ and Johnston [4] proved that this limit always exists by a simple average argument of Katona-Nemetz-Simonovits theorem [6]. They completely classified the Turán densities of $\{1,2\}$-graphs.

Theorem 1 (Lu and Johnston [4]). For any hypergraph $H$ with $R(H)=\{1,2\}$, we have

$$
\pi(H)= \begin{cases}2-\frac{1}{\mathcal{X}\left(H^{2}\right)-1} & \text { if } H^{2} \text { is not bipartite; } \\ \frac{5}{4} & \text { if } H^{2} \text { is bipartite and } \min \left\{k: \bar{P}_{2 k} \subseteq H\right\}=1 ; \\ \frac{9}{8} & \text { if } H^{2} \text { is bipartite and } \min \left\{k: \bar{P}_{2 k} \subseteq H\right\} \geqslant 2 ; \\ 1 & \text { if } H^{2} \text { is bipartite and } \bar{P}_{2 k} \nsubseteq H \text { for any } k \geqslant 1 .\end{cases}
$$

where $H^{2} \in H$ is the graph with all edges of cardinality 2. $\bar{P}_{2 k}$ is a closed path of length $2 k$, and $\mathcal{X}\left(H^{2}\right)$ is the chromatic number of $H^{2}$. 
It is trivial that $\pi(H) \leqslant|R(H)|$ and it is easy to see that $\pi(H) \geqslant|R(H)|-1$, since we can take an $(|R(H)|-1)$-complete hypergraph $K_{n}^{|R(H)|-1}$ without the appearance of $H$. We are interested in these $R$-graphs with the smallest Turán density.

Definition 1 (Degenerate hypergraphs). A hypergraph $H$ is called degenerate if $\pi(H)=$ $|R(H)|-1$.

What do the degenerate $R$-graphs look like? For the special case $R=\{r\}$, Erdös [3] showed that an $r$-uniform hypergraph $H$ is degenerate if and only if it is $r$-partite, that is, a sub-graph of a blow-up of a single edge of cardinality $r$. As a natural extension of a single edge, the chain $C^{R}$ for any set $R$ is degenerate. Thus every sub-graph of a blow-up of a chain is also degenerate. We say a degenerate $R$-graph is trivial if it is a sub-graph of a blow-up of the chain $C^{R}$. For $R=\{1,2\}$, by Theorem 1 all degenerate $\{1,2\}$-graphs are trivial. However, a nontrivial degenerate $\{2,3\}$-graph is found in [4]. It indicates that this question is more intrigue for other $R$-graphs.

In this paper, we will give a necessary and sufficient condition for the degenerate $\{1,3\}$-graphs. Given two graphs $G$ and $H$, we say $G$ is $H$-colorable if and only if there exists a hypergraph homomorphism $f$ from $G$ to $H$. (see Definition 3 ). And we have

Theorem 2. $A\{1,3\}$-hypergraph is degenerate if and only if it's $H_{5}^{\{1,3\}}$-colorable, where $H_{5}^{\{1,3\}}$ is a hypergraph with vertex set $V=[5]$ and edge set

$$
E=\{\{2\},\{3\},\{1,2,4\},\{1,3,5\},\{1,4,5\}\} .
$$

Using this non-trivial degenerate $\{1,3\}$-graph, we prove the following result.

Theorem 3. Let $R$ be a set of distinct positive integers with $|R| \geqslant 2$ and $R \neq\{1,2\}$. Then a non-trivial degenerate $R$-graph always exists.

We then continue to study the non-degenerate $\{1,3\}$-graphs. Let $K_{3}^{\bullet \bullet}$ be a $\{1,3\}$-graph with edges $\{\{1\},\{2\},\{1,2,3\}\}$, we have

Theorem 4. For any $K_{3}^{\bullet \bullet}$-colorable $\{1,3\}$-graph $H$, we have

1. if $K_{3}^{\bullet \bullet} \nsubseteq H, H$ must be $H_{5}^{\{1,3\}}$-colorable, then $\pi(H)=1$;

2. if $K_{3}^{\bullet \bullet} \subseteq H$, then $\pi(H)=\pi\left(K_{3}^{\bullet \bullet}\right)=1+\frac{\sqrt{3}}{18}$.

A result following Theorem 4 indicates a break for the Turán density of $\{1,3\}$-graphs:

Corollary 5. Let $\alpha$ be a real value in $\left[1, \frac{4}{9}+\frac{\sqrt{3}}{3}\right)$. For any $\{1,3\}$-graph $H$ with $\pi(H) \leqslant \alpha$, it must be the case that $\pi(H)=1$.

We also obtain the Turán densities of some 3-partite $\{1,3\}$-graphs, the results are shown in Section 4.

The paper is organized as follows. In section 2 we introduce some notations and lemmas for non-uniform hypergraphs. In section 3 we will prove the Theorem 2, Theorem 4 and Corollary 5. In section 4, we determine Turán densities of some 3 -partite $\{1,3\}$ graphs. In section 5, we prove Theorem 3. 


\section{Notation and lemmas}

In this section, we introduce some notations and lemmas for $R$-graphs and then for the $\{1,3\}$-graphs. We call an edge of cardinality $i$ as an $i$-edge, for each $i \in R$. For convenience, we call a vertex that forms a 1-edge as "black vertex", otherwise, "white vertex". We use notations of form $H_{n}^{\bullet}$ to represent a hypergraph on $n$ vertices that contains only one "black vertex", similarly, $H_{n}^{\bullet \bullet}$ represents a hypergraph on $n$ vertices that contains two "black vertices", and so on. To simplify our notations for $\{1,3\}$-graphs, we use form of $a b c$ to denote the edge $\{a, b, c\}$.

For a fixed set $R=\left\{k_{1}, k_{2}, \ldots, k_{r}\right\}$, with $\left(k_{1}<k_{2}<\ldots<k_{r}\right), R$-flag is an $R$-graph containing exactly one edge of each size. The chain $C^{R}$ is the special R-flag with the edge set $E\left(C^{R}\right)=\left\{\left[k_{1}\right],\left[k_{2}\right], \ldots,\left[k_{r}\right]\right\}$, where $\left[k_{i}\right]$ is the set of all positive integers from 1 to $k_{i}$ for each $i \in[r]$. For example, the chain $C^{\{1,3\}}=\{1,123\}$. For any $R$-flag $L$, we have $\pi(L)=|R|-1$ (see [4]). Thus the chain $C^{\{1,3\}}$ is a degenerate $\{1,3\}$-graph.

The following definitions and lemmas on non-uniform hypergraphs are generalized from uniform hypergraphs.

Definition 2 (Blow-up hypergraphs). [4] For any hypergraph $H$ on $n$ vertices and positive integers $s_{1}, s_{2}, \ldots, s_{n}$, the blow-up of $\mathrm{H}$ is a new hypergraph $(V, E)$, denoted by $H_{n}\left(s_{1}, s_{2}, \ldots, s_{n}\right)$, satisfying

- $V:=\bigsqcup_{i=1}^{n} V_{i}$, where $\left|V_{i}\right|=s_{i}$,

- $E:=\bigcup_{F \in E(H)} \prod_{i \in F} V_{i}$.

When $s_{1}=s_{2}=\ldots=s_{n}=s$, we simply write it as $H(s)$.

The blow-up operation does not change the Turán density.

Theorem 6 (Blow-up Families). [4] Let $\mathcal{H}$ be a finite family of hypergraphs and let $s \geqslant 2$. Then $\pi(\mathcal{H}(s))=\pi(\mathcal{H})$.

A direct corollary of Theorem 6 is the following result.

Theorem 7 (Squeeze Theorem). [4] Let $H$ be any hypergraph. If there exists a hypergraph $H^{\prime}$ and an integer $s \geqslant 2$ such that $H^{\prime} \subseteq H \subseteq H^{\prime}(s)$, then $\pi(H)=\pi\left(H^{\prime}\right)$.

It is easy to generalize the concepts of homomorphisms and $H$-coloring to general $R$-graphs.

Definition 3. Given two $R$-graphs $G$ and $H$, a hypergraph homomorphism is a vertex map $f: V(G) \rightarrow V(H)$ such that, if $\left\{v_{1}, \ldots, v_{r}\right\} \in E(G)$ then $\left\{f\left(v_{1}\right), \ldots, f\left(v_{r}\right)\right\} \in E(H)$, for all $r \in R$.

Definition 4 . A hypergraph $G$ is called $H$-colorable if and only if there exists a homomorphism from $G$ to $H$.

Note that, if there exists a homomorphism from $G$ to $H$, then $G$ is isomorphic to a sub-graph of a blow-up of $H$. Thus we have:

Lemma 8. If $G$ is $H$-colorable, then $\pi(G) \leqslant \pi(H)$. 


\section{$2.1 \quad R$-graphs with loops, blow-up, and Lagrangian}

A loop edge is a multiset of vertices. Sometimes we need to enlarge the concept of $R$ graphs to $R$-graphs with loops. For example, consider a $\{1,3\}$-graph $H_{1}$ with the edge set $\{x, x y y, y y y\}$. Here $x y y$ is a loop edge with vertex $x$ occurring once and vertex $y$ twice. In general, a loop edge $e=x_{1}^{m_{1}} \cdots x_{l}^{m_{l}}$ consists of $m_{1}$ copies of vertex $x_{1}, m_{2}$ copies of vertex $x_{2}$, and so on. For a loop edge $e=x_{1}^{m_{1}} \cdots x_{l}^{m_{l}}$, the cardinality of $e$ is $|e|=\sum_{i} m_{i}$. We also define a multinomial coefficient $c_{e}$ to be

$$
c_{e}:=\left(\begin{array}{c}
|e| \\
m_{1}, m_{2}, \ldots, m_{l}
\end{array}\right)=\frac{|e| !}{m_{1} ! m_{2} ! \cdots m_{l} !} .
$$

Definition 5. The polynomial form of an $R$-graph $H$ with loops on $n$ vertices, denoted by $\lambda(H, \vec{x})$ with $\vec{x}=\left(x_{1}, x_{2}, \ldots, x_{n}\right)$ is defined as

$$
\lambda(H, \vec{x}):=\sum_{e \in E(H)} c_{e} \prod_{i \in e} x_{i} .
$$

The Lagrangian of $H$, denoted by $\lambda(H)$, is the maximum value of the polynomial $\lambda(H, \vec{x})$ over the simplex $S_{n}=\left\{\left(x_{1}, x_{2}, \ldots, x_{n}\right) \in[0,1]^{n}: \sum_{i=1}^{n} x_{i}=1\right\}$.

For any $R$-graph $H$ (with possible loops), one can construct the family of $H$-colorable $R$-graph by blowing up $H$ in a certain way. The Lagrangian of $H$ is the maximum edge density of the $H$-colorable $R$-graphs that one can get in this way. This definition of Lagrangian is the same as the one in [5]; but differs from the classical Lagrangian for $r$-uniform hypergraphs such as in [8] by a constant multiplicative factor. This is not essential. This is a special case of more general Lagrangian of non-uniform hypergraphs introduced by Peng-Wu-Yao [9].

Construction A: Consider a $\{1,3\}$-graph (with loops) $H_{A}$ on two vertices $\{x, y\}$ with edges $\{x, x y y, y y y\}$. The polynomial form of $H_{A}$ is

$$
\lambda\left(H_{A}, \vec{x}\right)=x_{1}+3 x_{1} x_{2}^{2}+x_{2}^{3} .
$$

It can be shown that $\lambda(H, \vec{x})$ reaches the maximum $1+\frac{\sqrt{3}}{18}$ over the simplex $S_{2}=$ $\left\{\left(x_{1}, x_{2}\right) \in[0,1]^{2}, x_{1}+x_{2}=1\right\}$ at $x_{1}=\frac{1}{2}-\frac{\sqrt{3}}{6}$. Thus, we have

$$
\lambda\left(H_{A}\right)=1+\frac{\sqrt{3}}{18} \approx 1.096225 .
$$

A $\{1,3\}$-graph $G_{A}$ on $n$ vertices is generated by blowing-up $H_{A}$ as follows: set a vertex partition $V\left(G_{A}\right)=X \cup Y$ with $|X| \approx\left(\frac{1}{2}-\frac{\sqrt{3}}{6}\right) n$ such that all 1-edges are in $X$ (drawn by a black point), and all 3-edges are either formed by three vertices in $Y$ or by one vertex in $X$ plus two vertices in $Y$. In another words,

$$
E\left(G_{A}\right)=\left(\begin{array}{c}
X \\
1
\end{array}\right) \cup\left(\begin{array}{c}
X \\
1
\end{array}\right) \times\left(\begin{array}{c}
Y \\
2
\end{array}\right) \cup\left(\begin{array}{c}
Y \\
3
\end{array}\right)
$$


We have

$$
\begin{aligned}
h_{n}\left(G_{A}\right) & =\frac{|X|}{n}+\frac{|X|\left(\begin{array}{c}
|Y| \\
2
\end{array}\right)+\left(\begin{array}{c}
|Y| \\
3
\end{array}\right)}{\left(\begin{array}{c}
n \\
3
\end{array}\right)} \\
& =\lambda\left(H_{A}, \vec{x}\right)+o_{n}(1) . \\
& =\lambda\left(H_{A}\right)+o_{n}(1) .
\end{aligned}
$$

Here $\vec{x}=\left(\frac{|X|}{n}, \frac{|Y|}{n}\right)=\left(\frac{1}{2}-\frac{\sqrt{3}}{6}, \frac{1}{2}+\frac{\sqrt{3}}{6}\right)$.

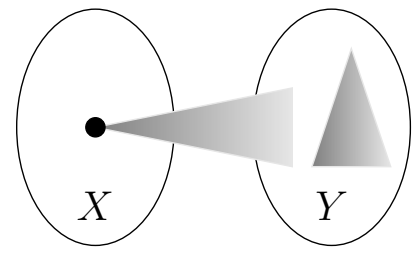

$$
G_{A}: \max h_{n}\left(G_{A}\right)=1+\frac{\sqrt{3}}{18}+o_{n}(1), \text { reached at }|X|=\left(\frac{1}{2}-\frac{\sqrt{3}}{6}\right) n
$$

Construction B: Let $H_{B}$ be a general $\{1,3\}$-graph on three vertices $\{a, b, c\}$ with the edge set $\{a, b, a b c\}$. We have

$$
\lambda\left(H_{B}, \vec{x}\right)=x_{1}+x_{2}+6 x_{1} x_{2} x_{3}
$$

It is easy to check

$$
\lambda\left(H_{B}\right)=\frac{4}{9}+\frac{\sqrt{3}}{3} \approx 1.021794714,
$$

which is reached at $x_{1}=x_{2}=\frac{1+\sqrt{3}}{6}$, and $x_{3}=\frac{2-\sqrt{3}}{3}$. A $\{1,3\}$-graph $G_{B}$ on $n$ vertices is generated by blowing-up $H_{B}$ as follows: set a vertex partition $V\left(G_{B}\right)=A \cup B \cup C$. All 1-edges are in $A$ and $B$ (drawn by black points), all 3-edges are formed by exactly one vertex in each partition. We have

$$
E\left(G_{B}\right)=\left(\begin{array}{c}
A \\
1
\end{array}\right) \cup\left(\begin{array}{c}
B \\
1
\end{array}\right) \cup\left(\begin{array}{c}
A \\
1
\end{array}\right) \times\left(\begin{array}{l}
B \\
1
\end{array}\right) \times\left(\begin{array}{l}
C \\
1
\end{array}\right) .
$$

Note that $G_{B}$ is $H_{B}$-colorable. Thus $h_{n}\left(G_{B}\right)=\lambda\left(H_{B}\right)+o_{n}(1)$.

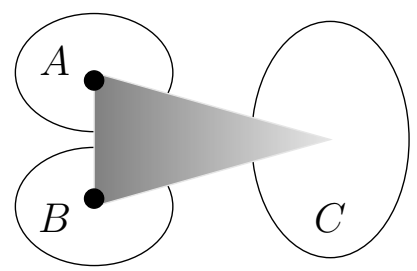

$G_{B}: \max h_{n}\left(G_{B}\right)=\frac{4}{9}+\frac{\sqrt{3}}{3}+o_{n}(1)$, reached at $|A|=|B|=\left(\frac{1+\sqrt{3}}{6}\right) n$. 


\section{$2.2 \quad$ Product of two $R$-graphs}

Let's define the product of $R$-graphs (with loops):

Definition 6 . For any two general $R$-graphs $H_{1}$ and $H_{2}$ with vertices set $V_{1}$ and $V_{2}$ respectively, we define the product of $H_{1}$ and $H_{2}$, which is denoted by $H_{1} \times H_{2}=(V, E)$, where

$$
V=V_{1} \times V_{2}, E=\cup_{r \in R} E\left(H_{1}^{r}\right) \times E\left(H_{2}^{r}\right),
$$

the $E\left(H_{i}^{r}\right)$ denotes the set of all edges of cardinality $r$ in $H_{i}$ for $i=1,2$. Here $E\left(H_{1}^{r}\right) \times$ $E\left(H_{2}^{r}\right)$ consists of all products of $e \times_{\sigma} f$, where $\sigma=(\sigma(1), \ldots, \sigma(r))$ takes over all permutations of $[r]$. For example, given $e=\left\{v_{1}, \ldots, v_{r}\right\} \in E\left(H_{1}\right), f=\left\{u_{1}, \ldots, u_{r}\right\} \in E\left(H_{2}\right)$, then $e \times_{\sigma} f=\left\{\left(v_{1}, u_{\sigma(1)}\right),\left(v_{2}, u_{\sigma(2)}\right), \ldots,\left(v_{r}, u_{\sigma(r)}\right)\right\}$ is an edge in $E\left(H_{1}^{r}\right) \times E\left(H_{2}^{r}\right)$.

Example 1. The product of two $\{1,3\}$-graphs $H_{A}$ and $H_{B}$ is given below. Let ax stand for $(a, x)$, similar for other labels, then the vertex set is $V\left(H_{A} \times H_{B}\right)=\{a x, a y, b x, b y, c x, c y\}$ and the edge set is

$$
E\left(H_{A} \times H_{B}\right)=\{\{a x\},\{b x\},\{c y, b x, a y\},\{c y, a y, b y\},\{c y, b y, a x\},\{c x, a y, b y\}\} .
$$

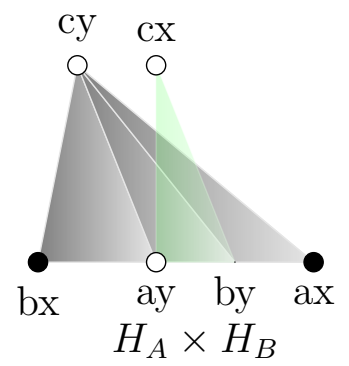

Lemma 9. For any two $R$-graphs $H_{1}$ and $H_{2}$, if hypergraph $\mathrm{H}$ is $H_{1}$ and $H_{2}$-colorable, then it's $\left(H_{1} \times H_{2}\right)$-colorable.

Proof. By definition, there exist two graph homomorphisms $f_{1}: V(H) \mapsto V\left(H_{1}\right)$ and $f_{2}: V(H) \mapsto V\left(H_{2}\right)$. Note that $H$ could be an $R$-graph. Then for any $r \in R$, if edge $e=\left\{v_{1}, \ldots, v_{r}\right\} \in E(H)$, we have

$$
f_{1}(e)=\left\{f_{1}\left(v_{1}\right), \ldots, f_{1}\left(v_{r}\right)\right\} \in E\left(H_{1}\right)
$$

and

$$
f_{2}(e)=\left\{f_{2}\left(v_{1}\right), \ldots, f_{2}\left(v_{r}\right)\right\} \in E\left(H_{2}\right) .
$$

Define a map $f:=f_{1} \times f_{2}$ from $V(H)$ to $V\left(H_{1}\right) \times V\left(H_{2}\right)$, such that $f(v)=\left(f_{1}(v), f_{2}(v)\right)$. Then we have

$$
f(e)=\left\{\left(f_{1}\left(v_{1}\right), f_{2}\left(v_{1}\right)\right), \ldots,\left(f_{1}\left(v_{r}\right), f_{2}\left(v_{r}\right)\right)\right\} \in f_{1}(e) \times f_{2}(e) \subseteq E\left(H_{1} \times H_{2}\right) .
$$

Thus the map $f$ takes edges in $H$ to edges in $H_{1} \times H_{2}$, it is a graph homomorphism. Therefore, $H$ is $\left(H_{1} \times H_{2}\right)$-colorable. 


\section{Proof of Theorem 2 and Theorem 4}

\subsection{Proof of Theorem 2}

For $\{1,3\}$-graph $H$, we have $1 \leqslant \pi(H) \leqslant 2$. Observe that the product of two general $R$-graphs could be an $R$-graph. This is very useful in determining the Turán density. A degenerate $R$-graph $H$ must be $G$-colorable for any $R$-graph $G$ with $\lambda(G)>1$. By Lemma 9 , it must be colorable by the product of these $R$-graphs. In this section, we will characterize the degenerate $\{1,3\}$-graph. Let's consider $H_{5}^{\{1,3\}}$, the 4 -vertex $\{1,3\}$-graph with edge set $\{2,3,124,135,145\}$.

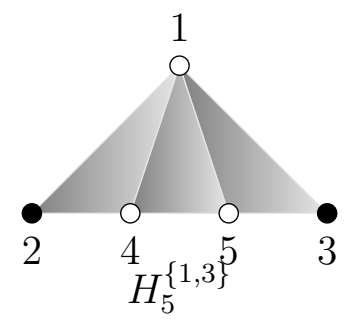

We first prove the following lemma.

Lemma 10. Any degenerate $\{1,3\}$-graph is $H_{5}^{\{1,3\}}$-colorable.

Proof. Observe that a degenerate $\{1,3\}$-graph $H$ must be contained in $G_{A}$ and $G_{B}$. Equivalently, $H$ is both $H_{A}$ and $H_{B}$-colorable, then it must be colorable by the product $H_{A} \times H_{B}$. We define a map $f: V\left(H_{A} \times H_{B}\right) \rightarrow[5]$ such that: $f(c x)=f(c y)=1$, $f(b x)=2, f(a x)=3, f(a y)=4, f(b y)=5$. Obviously, $f$ is a graph homomorphism from $H_{A} \times H_{B}$ to $H_{5}^{\{1,3\}}$. The result follows.

Let $K_{3}^{\bullet \bullet}$ be a $\{1,3\}$-graph on 3 vertices with edges $\{1,2,123\}$, and $G_{4}^{\bullet}$ be a $\{1,3\}$-graph on 4 vertices with edges $\{1,123,134,234\}$.

Remark $11 . K_{3}^{\bullet \bullet}$ is not contained in $G_{A}$ whose edge density reaches $1+\frac{\sqrt{3}}{18}$, and $G_{4}^{\bullet}$ is not contained in $G_{B}$ whose edge density reaches $\frac{4}{9}+\frac{\sqrt{3}}{3}$. Thus both $K_{3}^{\bullet \bullet}$ and $G_{4}^{\bullet}$ are non-degenerate $\{1,3\}$-graphs.

Lemma 12. $\pi\left(\left\{K_{3}^{\bullet \bullet}, G_{4}^{\bullet}\right\}\right)=1$.

Proof. For any positive integer $n$, let $G$ be a $\left\{K_{3}^{\bullet \bullet}, G_{4}^{\bullet}\right\}$-free $\{1,3\}$-graph on $n$ vertices. Denote $S$ as the set of all 1-edges of $G$, i.e. $S=\{v \in V(G):\{v\} \in E(G)\}$, and let $|S|=x n$ for some $x \in(0,1)$. Let $\bar{S}$ be the complement of $S$, i.e. $\bar{S}=V(G) \backslash S$, then $|\bar{S}|=(1-x) n$.

Denote $E\left(G^{3}\right)$ as the set of all 3-edges of $G$. To forbidden $K_{3}^{\bullet \bullet}$, there is at most one black vertex in any 3 -edges of $G$, thus we have

$$
E\left(G^{3}\right) \subseteq\left(\begin{array}{l}
S \\
1
\end{array}\right) \times\left(\begin{array}{l}
\bar{S} \\
2
\end{array}\right) \cup\left(\begin{array}{l}
\bar{S} \\
3
\end{array}\right)
$$


We consider the 3 -edges of $G$ in edge set $\left(\begin{array}{l}S \\ 1\end{array}\right) \times\left(\begin{array}{l}\bar{S} \\ 2\end{array}\right)$. Define $y$ as the average edge density of such 3 -edges in $G$. Thus

$$
y=\frac{\left|E\left(G^{3}\right) \cap\left(S \times\left(\begin{array}{c}
\bar{S} \\
2
\end{array}\right)\right)\right|}{|S| \times\left(\begin{array}{c}
|\bar{S}| \\
2
\end{array}\right)} .
$$

Note that there exists one vertex $s_{0} \in S$ such that $\left|C\left(s_{0}\right)\right| \geqslant y \times\left(\begin{array}{c}|\bar{S}| \\ 2\end{array}\right)$, where $C\left(s_{0}\right)$ is the set of 3 -edges that contain the black vertex $s_{0}$. For any vertex $u \in \bar{S}$, define

$$
W_{u}:=\left\{v \in \bar{S} \mid s_{0} u v \in E(G)\right\}
$$

We then have

$$
\sum_{u \in \bar{S}}\left|W_{u}\right| \leqslant|\bar{S}| \times(|\bar{S}|-1)
$$

and

$$
\left|C\left(s_{0}\right)\right|=\frac{1}{2} \sum_{u \in \bar{S}}\left|W_{u}\right|
$$

which implies

$$
\sum_{u \in \bar{S}}\left|W_{u}\right| \geqslant 2 y \times\left(\begin{array}{c}
|\bar{S}| \\
2
\end{array}\right) .
$$

To forbidden $G_{4}^{\bullet}$, if $s_{0} u v, s_{0} u k \in E(G)$, then $u v k \notin E(G)$. Since for each $u \in \bar{S}$, there are $\left(\begin{array}{c}\left|W_{u}\right| \\ 2\end{array}\right)$ pair of vertices each can form a 3-edge with $u$, we need to remove these edges in $\left(\begin{array}{l}\bar{S} \\ 3\end{array}\right)$. Let $N$ be the number of 3-edges in $\left(\begin{array}{l}\bar{S} \\ 3\end{array}\right)$ but not in $G$, by Cauchy-Schwarz inequality, we have

$$
\begin{aligned}
N & \geqslant \frac{1}{3} \sum_{u \in \bar{S}}\left(\begin{array}{c}
\left|W_{u}\right| \\
2
\end{array}\right) \\
& \geqslant \frac{1}{6} \frac{1}{|\bar{S}|}\left(\sum_{u \in \bar{S}}\left|W_{u}\right|\right)^{2}-\frac{1}{6} \sum_{u \in \bar{S}}\left|W_{u}\right| \\
& \geqslant \frac{1}{6} y^{2}|\bar{S}|^{3}-\frac{1}{6}|\bar{S}| \times(|\bar{S}|-1) .
\end{aligned}
$$

Thus we have

$$
h_{n}(G) \leqslant x+y \times 3 x \times(1-x)^{2}+(1-x)^{3}-y^{2}(1-x)^{3}+o_{n}(1) .
$$

When $x \leqslant \frac{2}{5}$, the above expression reaches the maximum value when $y=\frac{3}{2} \frac{x}{1-x} \leqslant 1$. When $x \geqslant \frac{2}{5}$, the above expression reaches the maximum value when $y=1$. Thus we obtain

$$
h_{n}(G) \leqslant \begin{cases}x+(1-x)^{3}+\frac{9}{4} x^{2}(1-x) & \text { for } x \leqslant \frac{2}{5} \\ x+3 x(1-x)^{2} & \text { for } x \geqslant \frac{2}{5}\end{cases}
$$


When $x \leqslant \frac{2}{5}$, by solving $f(x)=x+(1-x)^{3}+\frac{9}{4} x^{2}(1-x) \leqslant 1$, we get $x \leqslant \frac{8}{13}$. This always holds since $x \leqslant \frac{2}{5} \leqslant \frac{8}{13}$. When $x \geqslant \frac{2}{5}, g(x)=x+3 x(1-x)^{2} \leqslant 1$ is equivalent to $3 x(1-x) \leqslant 1$ which is always true. Thus in both cases, we have $h_{n}(G) \leqslant 1+o_{n}(1)$, implies that $\pi\left(\left\{K_{3}^{\bullet \bullet}, G_{4}^{\bullet}\right\}\right)=\lim _{n \rightarrow \infty} h_{n}(G)=1$. The proof is complete.

Proof of Theorem 2. Note that $H_{5}^{\{1,3\}}$ is $K_{3}^{\bullet \bullet}$ and $G_{4}^{\bullet}$-colorable respectively, we have

$$
\pi\left(H_{5}^{\{1,3\}}\right) \leqslant \pi\left(\left\{K_{3}^{\bullet \bullet}, G_{4}^{\bullet}\right\}\right) .
$$

By Lemma 10 and Lemma 12, the result follows.

\subsection{Proof of Theorem 4}

In this subsection, we will consider the non-degenerate $\{1,3\}$-graphs. In particularly, we consider the non-degenerate 3 -partite $\{1,3\}$-graphs. A hypergraph is called 3-partite if its vertex set $V$ can be partitioned into 3 different classes $V_{1}, V_{2}, V_{3}$ such that every edge

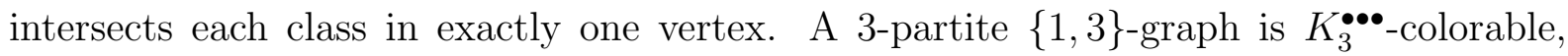
where $K_{3}^{\bullet \bullet \bullet}$ is a $\{1,3\}$-graph on 3 vertices with edge set $\{1,2,3,123\}$.

So far we know that the chain $C^{\{1,3\}}=\{1,123\}$ is 3-partite and it is degenerate, while a slightly larger 3-partite $\{1,3\}$-graph $K_{3}^{\bullet \bullet}=\{1,2,123\}$ is not degenerate. We have $\pi\left(K_{3}^{\bullet \bullet}\right) \geqslant 1+\frac{\sqrt{3}}{18}$ since it's not contained in the $G_{A}$. Now we are ready to prove Theorem 4 and Corollary 5.

Proof of Theorem 4. For Item 1: For graph $H$ in the assumption, there exists a vertex partition $V(H)=V_{1} \cup V_{2} \cup V_{3}$ so that $H$ is 3-partite and the level-graph $H^{1}$ only appears in at most two vertex partitions (say $V_{2}$ and $V_{3}$ ). Since $H$ does not contain $K_{3}^{\bullet \bullet}$ as subgraph, then each edge of the level-graph $H^{3}$ can only intersect one vertex in $V_{1}$, plus one white (black) vertex in $V_{2}$ and one black (white) vertex in $V_{3}$, or intersect one vertex in $V_{1}$ plus two white vertices in $V_{2}$ and $V_{3}$. Let $f$ be a map such that $f(v)=1$ if $v \in V_{1}$, $f(v)=2$ if $v$ is a black vertex in $V_{2}, f(v)=5$ if $v$ is a white vertex in $V_{2}$, and $f(v)=3$ if $v$ is a black vertex in $V_{3}, f(v)=4$ if $v$ is a white vertex in $V_{3}$. One can check that $f$ is a hypergraph homomorphism from $H$ to $H_{5}^{\{1,3\}}$. Thus $H$ is $H_{5}^{\{1,3\}}$-colorable, we have $\pi(H)=1$.

For Item 2: Since $K_{3}^{\bullet \bullet} \subseteq H \subseteq K_{3}^{\bullet \bullet}(s)$ for $s \geqslant 2$, by Theorem 7 , we have $\pi(H)=$ $\pi\left(K_{3}^{\bullet \bullet}\right)$. For any $K_{3}^{\bullet \bullet}$-free $\{1,3\}$-graph $G$ on $n$ vertices, let $X$ be the set of all 1 -edges in $G$, and $Y \subseteq V(G)$ be the complement of $X$. On one hand, since $G$ is $K_{3}^{\bullet \bullet}$-free, there is no 3-edge of form $\left(\begin{array}{l}X \\ 3\end{array}\right)$ or form $\left(\begin{array}{l}X \\ 2\end{array}\right) \times\left(\begin{array}{l}Y \\ 1\end{array}\right)$. Thus $G$ is $H_{A}$-colorable. Therefore $\lim _{n \rightarrow \infty} h_{n}(G) \leqslant \lambda\left(H_{A}\right)=1+\frac{\sqrt{3}}{18}$. On the other hand, the construction $G_{A}$ is $K_{3}^{\bullet \bullet}$-free. We have $\pi\left(K_{3}^{\bullet \bullet}\right) \geqslant \lambda\left(H_{A}\right)=1+\frac{\sqrt{3}}{18}$. Thus, $\pi(H)=\pi\left(K_{3}^{\bullet \bullet}\right)=1+\frac{\sqrt{3}}{18}$.

Proof of Corollary 5: Let $H$ be any $\{1,3\}$-graph with $\pi(H)<\lambda\left(H_{B}\right)=\frac{4}{9}+\frac{\sqrt{3}}{3}$. Then $H$ must be $H_{B}$-colorable, hence $K_{3}^{\bullet \bullet-c o l o r a b l e . ~ B y ~ I t e m ~} 2$ of Theorem $4, \pi(H)$ is either 1 or $1+\frac{\sqrt{3}}{18}$, thus we must have $\pi(H)=1$. 


\section{The 3-partite $\{1,3\}$-graphs}

In previous section, all $\{1,3\}$-graphs we studied are 3 -partite. In this section, we continue to study the Turán densities of 3-partite $\{1,3\}$-graphs.

Lemma 13. Let $H$ be a 3-partite $\{1,3\}$-graph such that $K_{3}^{\bullet \bullet \bullet} \subseteq H$, where $K_{3}^{\bullet \bullet \bullet}=$ $\{1,2,3,123\}$. Then $\pi(H)=1+\frac{2 \sqrt{3}}{9}$.

Proof. Since any 3-partite $\{1,3\}$-graph is $K_{3}^{\bullet \bullet \bullet-c o l o r a b l e, ~ t h e n ~} \pi(H)=\pi\left(K_{3}^{\bullet \bullet \bullet}\right)$. We will prove $\pi\left(K_{3}^{\bullet \bullet \bullet}\right)=1+\frac{2 \sqrt{3}}{9}$.

On one hand, consider an extremal $K_{3}^{\bullet \bullet \bullet-f r e e ~}\{1,3\}$-graph $G_{n}$. Let $X$ be the set of vertices of 1-edges in $G_{n}$. Projecting all the vertices in $X$ into a single vertex $x$ and all the vertices not in $X$ into a single vertex $y$, we get an $\{1,3\}$-graph (with loops) $H_{C}$ : where $E\left(H_{C}\right)=\{x, x x y, x y y, y y y\}$. This projection is a hypergraph homomorphism from $G_{n}$ to $H_{C}$ since $G_{n}$ is $K_{3}^{\bullet \bullet \bullet-f r e e . ~ T h u s ~} G_{n}$ is $H_{C}$-colorable. In particular, we have

$$
\pi\left(K_{3}^{\bullet \bullet \bullet)}=\lim _{n \rightarrow \infty} h_{n}\left(G_{n}\right) \leqslant \lambda\left(H_{C}\right)=1+\frac{2 \sqrt{3}}{9} .\right.
$$

On the other hand, any blow-up of $H_{C}$ does not contain the sub-graph $K_{3}^{\bullet \bullet \bullet}$. The following blow-up graph $G_{C}$ has the maximal edge density $1+\frac{2 \sqrt{3}}{9}$ :

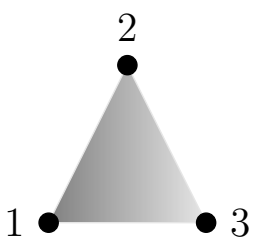

$K_{3}^{\bullet \bullet \bullet}$

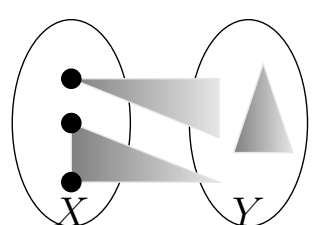

$G_{C}: h_{n}\left(G_{C}\right)=1+\frac{2 \sqrt{3}}{9}+o_{n}(1)$, at $|X|=\frac{\sqrt{3}}{3} n$.

Hence, $\pi(H)=\pi\left(K_{3}^{\bullet \bullet \bullet)}=\lambda\left(H_{C}\right)=1+\frac{2 \sqrt{3}}{9}\right.$.

Let us restrict $H$ to be 3 -partite but containing no $K_{3}^{\bullet \bullet \bullet}$ as sub-graph. One can check such $H$ must be $H_{6}^{\{1,3\}}$-colorable, where

$$
H_{6}^{\{1,3\}}=\{1,2,3,124,145,135,236,246,356,456\} .
$$

$H_{6}^{\{1,3\}}$ is not contained in $G_{D}$ with maximal edge density $\frac{4}{3}$.
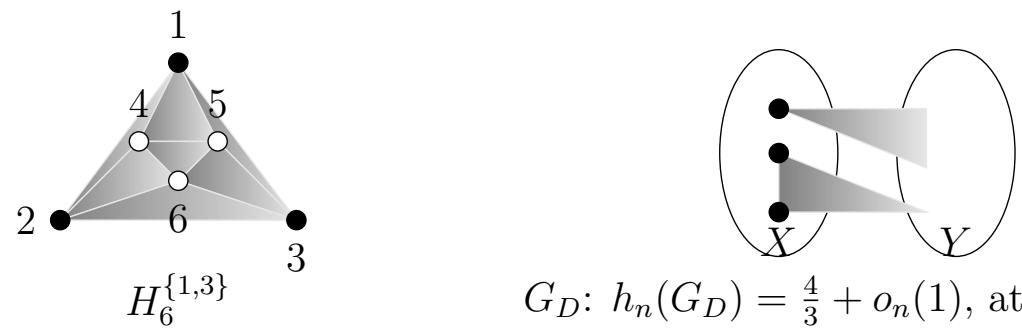

$G_{D}: h_{n}\left(G_{D}\right)=\frac{4}{3}+o_{n}(1)$, at $|X|=\frac{2}{3} n$. 
So far we couldn't determine the upper bound of $\pi\left(H_{6}^{\{1,3\}}\right)$. We leave this open. Let's turn our attention to the sub-graphs of $H_{6}^{\{1,3\}}$ and we aim to determine their Turán densities. We first consider two sub-graphs of $H_{6}^{\{1,3\}}: H_{5}^{*}=\{1,2,3,124,145,135\}$ and $H_{6}^{*}=$ $\{1,2,3,124,135,236\}$. For both of them, the Turán density is greater than $1+\frac{\sqrt{3}}{9}$, since they are not contained in $G_{E}$ and $G_{F}$ respectively $\left(\lim _{n \rightarrow \infty} h_{n}\left(G_{E}\right)=\lim _{n \rightarrow \infty} h_{n}\left(G_{F}\right)\right.$ ).
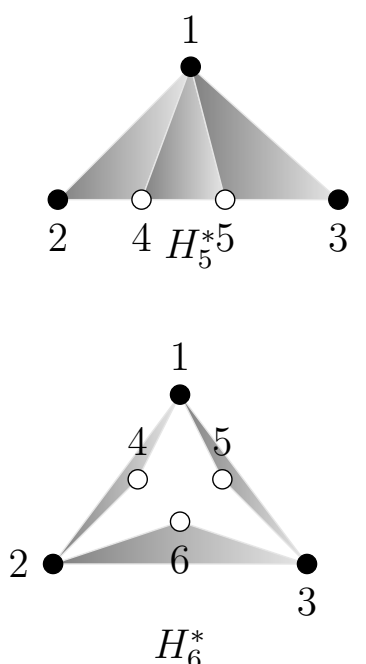

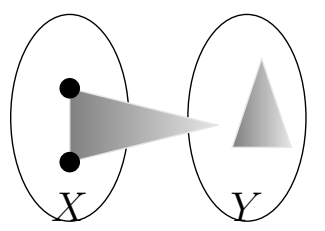

$$
G_{E}: h_{n}\left(G_{E}\right)=\frac{9+\sqrt{3}}{9}+o_{n}(1), \text { at }|X|=\left(\frac{3+\sqrt{3}}{6}\right) n \text {. }
$$

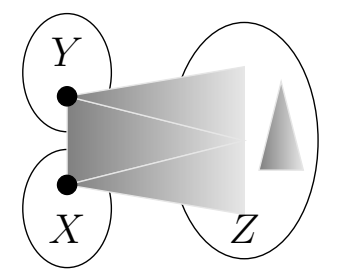

$$
G_{F}: h_{n}\left(G_{F}\right)=\frac{9+\sqrt{3}}{9}+o_{n}(1), \text { at }|X|=|Y|=\left(\frac{3-\sqrt{3}}{6}\right) n \text {. }
$$

To calculate the upper bounds of $\pi\left(H_{5}^{*}\right)$, we need the following lemma.

Lemma 14. Let $H_{4}^{\bullet \bullet}=\{1,2,123,124,134\}$, then $\pi\left(\left\{K_{3}^{\bullet \bullet \bullet}, H_{4}^{\bullet \bullet}\right\}\right)=1+\frac{\sqrt{3}}{9}$.

Proof. To see the lower bound, observe that both $H_{4}^{\bullet \bullet}$ and $K_{3}^{\bullet \bullet \bullet}$ are not contained in $G_{E}$.

To see the upper bound, let $G$ represent a $\left\{K_{3}^{\bullet \bullet \bullet}, H_{4}^{\bullet \bullet}\right\}$-free graph on $n$ vertices, let $X \subseteq V(G)$ be the set of all 1-edges of $G,|X|=x n$ for some real $x \in(0,1)$, and let $Y=V(G) \backslash X$, then $|Y|=(1-x) n$. To forbidden $K_{3}^{\bullet \bullet \bullet}$, there is no 3-edge of form $\left(\begin{array}{l}X \\ 3\end{array}\right)$. Let $y$ be the density of 3 -edges in $G$ among all edges of form $\left(\begin{array}{c}X \\ 2\end{array}\right) \times\left(\begin{array}{l}Y \\ 1\end{array}\right)$. For any pair of vertices $(i, j)$ in $X$, denote $d_{i j}$ as the number of vertices $k \in Y$ so that $\{i j k\} \in E(G)$. Then

$$
y=\frac{\sum_{(i, j) \in\left(\begin{array}{c}
X \\
2
\end{array}\right)} d_{i j}}{\left(\begin{array}{c}
|X| \\
2
\end{array}\right) \times\left(\begin{array}{c}
|Y| \\
1
\end{array}\right)} .
$$

To forbidden $H_{4}^{\bullet \bullet}$, for each pair of vertices $(i, j) \in\left(\begin{array}{c}X \\ 2\end{array}\right)$, if $i j k$ and $i j l$ are in $E(G)$, neither $k l i$ nor $k l j$ can be contained in $E(G)$. Thus for every pair of $\{i, j\}$, the number of 3-edges not shown in $G$ is at least $2\left(\begin{array}{c}d_{i j} \\ 2\end{array}\right)$. Let $M$ be the total number of 3-edges of form $\left(\begin{array}{l}X \\ 1\end{array}\right) \times\left(\begin{array}{l}Y \\ 2\end{array}\right)$ not shown in $G$, then by Cauchy-Schwarz inequality, we have

$$
M \geqslant \frac{\sum_{i, j \in\left(\begin{array}{c}
X \\
2
\end{array}\right)} 2\left(\begin{array}{c}
d_{i j} \\
2
\end{array}\right)}{|X|}
$$




$$
\begin{aligned}
& \geqslant \frac{\left(\sum_{i, j \in X} d_{i j}\right)^{2}}{\left(\begin{array}{c}
x n \\
2
\end{array}\right)(x n)}-\frac{\sum_{i, j \in X} d_{i j}}{x n} \\
& \geqslant \frac{1}{2} y^{2} x(1-x)^{2} n^{3}-\frac{1}{2} y x(1-x) n^{2} .
\end{aligned}
$$

Thus

$$
h_{n}(G) \leqslant x+(1-x)^{3}+3 x^{2}(1-x) y+3 x(1-x)^{2}-3 x(1-x)^{2} y^{2}+o_{n}(1) .
$$

A simple calculation can show that $h_{n}(G)$ achieves maximum value at $y=1$, which implies that for any positive integer $n$, any extremal $\left\{K_{3}^{\bullet \bullet \bullet}, H_{4}^{\bullet \bullet}\right\}$-free $\{1,3\}$-graph is $H_{E^{-}}$-colorable where $E\left(H_{E}\right)=\{x, x x y, y y y\}$. Therefore, $\pi\left(\left\{K_{3}^{\bullet \bullet \bullet}, H_{4}^{\bullet \bullet}\right\}\right)=\lim _{n \rightarrow \infty} h_{n}\left(G_{n}\right) \leqslant \lambda\left(H_{E}\right)=$ $1+\frac{\sqrt{3}}{9}$. The result follows.

Lemma 15. $\pi\left(H_{5}^{*}\right)=1+\frac{\sqrt{3}}{9}$.

Proof. On one hand, $H_{5}^{*}$ is not contained in $G_{E}$, then $\pi\left(H_{5}^{*}\right) \geqslant 1+\frac{\sqrt{3}}{9}$. On the other hand, $H_{5}^{*}$ is $K_{3}^{\bullet \bullet \bullet}$ and $H_{4}^{\bullet \bullet-c o l o r a b l e, ~ t h u s ~} \pi\left(H_{5}^{*}\right) \leqslant \pi\left(\left\{K_{3}^{\bullet \bullet \bullet}, H_{4}^{\bullet \bullet}\right\}\right) \leqslant 1+\frac{\sqrt{3}}{9}$. The result follows.

Corollary 16. The proper sub-graphs of $H_{5}^{*}$ can be classified to two different sets: either the sub-graph contains $K_{3}^{\bullet \bullet}$ and is $K_{3}^{\bullet \bullet-c o l o r a b l e, ~ i n ~ t h i s ~ c a s e ~ t h e ~ T u r a ́ n ~ d e n s i t y ~ i s ~} 1+\frac{\sqrt{3}}{18}$; or the sub-graph does not contain $K_{3}^{\bullet \bullet}$, then it is $H_{5}^{\{1,3\}}$-colorable, in this case the Turán density is 1.

To calculate the upper bounds of $\pi\left(H_{6}^{*}\right)$, we need the following lemma.

Lemma 17. Let $H_{4}^{\bullet \bullet \bullet}=\{1,2,3,124,134,234\}$, then $\pi\left(\left\{K_{3}^{\bullet \bullet \bullet}, H_{4}^{\bullet \bullet \bullet}\right\}\right)=1+\frac{\sqrt{3}}{9}$.

Proof. To see the lower bound, observe that both $K_{3}^{\bullet \bullet \bullet}$ and $H_{4}^{\bullet \bullet \bullet}$ are not contained in $G_{F}$. To see the upper bound, let $G$ represent a $\left\{K_{3}^{\bullet \bullet \bullet}, H_{4}^{\bullet \bullet \bullet}\right\}$-free graph on $n$ vertices, let $X \subseteq V(G)$ be the set of all 1-edges of $G,|X|=x n$ for some real $x \in(0,1)$, let $Y=V(G) \backslash X$, then $|Y|=(1-x) n$. To forbidden $K_{3}^{\bullet \bullet \bullet}$, there is no 3-edge of form $\left(\begin{array}{l}X \\ 3\end{array}\right)$.

Let $y$ be the density of 3-edges in $G$ among all edges of form $\left(\begin{array}{c}X \\ 2\end{array}\right) \times\left(\begin{array}{l}Y \\ 1\end{array}\right)$. For each $i \in Y$, let $D_{i}=\left\{\{j, k\} \in\left(\begin{array}{c}X \\ 2\end{array}\right) \mid i j k \in E(G)\right\}$, denote $d_{i}=\left|D_{i}\right|$. Then

$$
y=\frac{\sum_{i \in Y} d_{i}}{\left(\begin{array}{c}
|X| \\
2
\end{array}\right) \times\left(\begin{array}{c}
|Y| \\
1
\end{array}\right)} .
$$

Suppose $y>\frac{1}{2}$, then there exists $i \in Y$, such that $d_{i}>\frac{1}{2}\left(\begin{array}{c}|X| \\ 2\end{array}\right)$. By the fact that the Turán density of a triangle-free graph is $\frac{1}{2}$, there must exist a triple $\{j, k, l\} \in\left(\begin{array}{l}X \\ 3\end{array}\right)$ such that $\{i j k, i j l, i k l\} \subseteq E(G)$, which is a copy of $H_{4}^{\bullet \bullet \bullet}$, a contradiction. Thus we have $y \leqslant \frac{1}{2}$. Note that the existence of 3-edge of form $\left(\begin{array}{c}Y \\ 3\end{array}\right)$ or $\left(\begin{array}{c}X \\ 1\end{array}\right) \times\left(\begin{array}{c}Y \\ 2\end{array}\right)$ does not result in an occurrence of $H_{4}^{\bullet \bullet \bullet}$ or $K_{3}^{\bullet \bullet \bullet}$ in $G$. Thus we can take all such edges. then

$$
h_{n}(G) \leqslant x+(1-x)^{3}+\frac{3}{2} x^{2}(1-x)+3 x(1-x)^{2}+o_{n}(1)
$$


which achieves the maximum $1+\frac{\sqrt{3}}{9}$ at $x=1-\frac{\sqrt{3}}{3}$.

Hence, we have $\pi\left(\left\{K_{3}^{\bullet \bullet \bullet}, H_{4}^{\bullet \bullet \bullet}\right\}\right)=\lim _{n \rightarrow \infty} h_{n}\left(G_{n}\right) \leqslant 1+\frac{\sqrt{3}}{9}$. The result follows.

Lemma 18. $\pi\left(H_{6}^{*}\right)=1+\frac{\sqrt{3}}{9}$.

Proof. On one hand, $H_{6}^{*}$ is not contained in $G_{F}$, then $\pi\left(H_{6}^{*}\right) \geqslant 1+\frac{\sqrt{3}}{9}$. On the other hand, $H_{6}^{*}$ is $K_{3}^{\bullet \bullet \bullet}$ and $H_{4}^{\bullet \bullet \bullet-c o l o r a b l e, ~ t h u s ~} \pi\left(H_{6}^{*}\right) \leqslant \pi\left(\left\{K_{3}^{\bullet \bullet \bullet}, H_{4}^{\bullet \bullet \bullet}\right\}\right) \leqslant 1+\frac{\sqrt{3}}{9}$. The result follows.

Since we are considering all sub-graphs of $H_{6}^{\{1,3\}}$, we start this by looking at the larger sub-graphs then the smaller sub-graphs. Using above two lemmas, we are able to determine the Turán density for a list of sub-graphs of $H_{6}^{\{1,3\}}$. Now we let $H$ be a sub-graph of $H_{6}^{\{1,3\}}$, we have:

1. If $H$ is $K_{3}^{\bullet \bullet-c o l o r a b l e, ~ t h u s ~} \pi(H)=1$ or $\pi(H)=1+\frac{\sqrt{3}}{18}$.

2. If $H$ is not above case, then $H$ must contain all 1-edges: 1,2,3, and none of them is isolated. Then we have several different cases:

(a) Suppose $H$ is obtained from $H_{6}^{\{1,3\}}$ by removing one 3-edge consisting of two black vertices and one white vertex (say 236 or equivalence), then one can check $H$ is $H_{5}^{*}$-colorable. Note that $H_{5}^{*} \subseteq H$, by Lemmas 15 , we have $\pi(H)=1+\frac{\sqrt{3}}{9}$. Similarly, for any sub-graph $H^{\prime}$ of $H$, if $H^{\prime}$ contains $H_{5}^{*}$ or a $H_{5}^{*}$-colorable graph as sub-graph, then $\pi\left(H^{\prime}\right)=1+\frac{\sqrt{3}}{9}$. If $H^{\prime}$ is not above case, by trial and error, there is only one situation: $H^{\prime}$ contains the following sub-graph $H^{*}$ (or its equivalence) which is not contained in $G_{E}$. Thus $\pi\left(H^{\prime}\right)=1+\frac{\sqrt{3}}{9}$ :

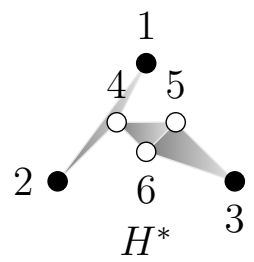

(b) Let $H$ be sub-graph of $H_{6}^{\{1,3\}}$ by removing edges 145, 246, 456 and 356, the resulting graph is $H_{6}^{*}$. By Lemma $18, \pi(H)=\pi\left(H_{6}^{*}\right)=1+\frac{\sqrt{3}}{9}$.

3. The following graphs $H_{6}^{\{1,3\}}, H_{6}^{a}, H_{6}^{b}, H_{6}^{c}, H_{6}^{d}$ and $H_{6}^{e}$ are unsolved. We conjecture that the extremal configuration of $H_{6}^{\{1,3\}}$ and $H_{6}^{b}$ is Construction $G_{D}$ : thus we conjecture $\pi\left(H_{6}^{\{1,3\}}\right)=\pi\left(H_{6}^{b}\right)=\frac{4}{3}$.
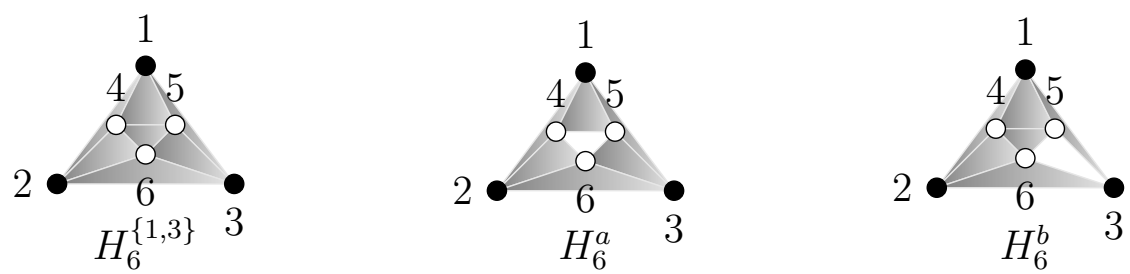

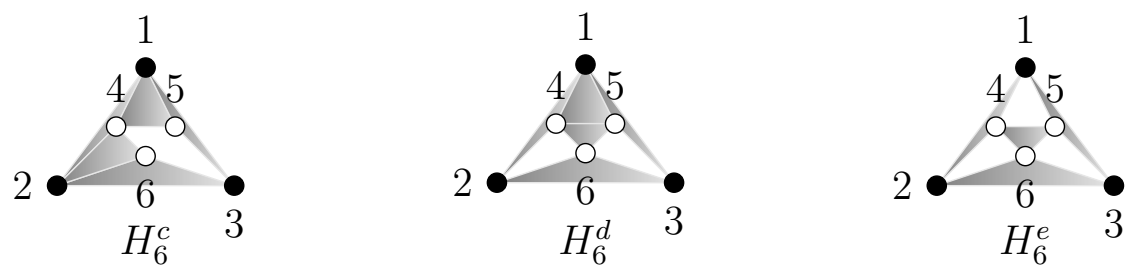

\section{Non-trivial degenerate $R$-graphs}

Recall that a degenerate $R$-graph $H$ is trivial if it is contained in a blow-up of the chain $C^{R}$, otherwise, we say $H$ is non-trivial. In this section, we will show that except the case $R=\{1,2\}$, there always exist non-trivial degenerate $R$-graphs for any finite set $R$ of at least two distinct positive integers. We will use the suspension operations on hypergraphs.

Definition 7. [4] The suspension of a hypergraph $H$, denoted by $S(H)$, is the hypergraph with $V=V(H) \cup\{v\}$ where $\{v\}$ is a new vertex not in $V(H)$, and the edge set $E=$ $\{e \cup\{v\}: e \in E(H)\}$. We write $S^{t}(H)$ to denote the hypergraph obtained by iterating the suspension operation $t$-times, i.e. $S^{2}(H)=S(S(H))$ and $S^{3}(H)=S(S(S(H)))$, etc.

The relationship between $\pi(H)$ and $\pi(S(H))$ was investigated in [4].

Proposition 1. [4] For any family of hypergraphs $\mathcal{H}$ we have that $\pi(S(\mathcal{H})) \leqslant \pi(\mathcal{H})$.

Given a general set $R$ and positive integer $t$, we denote $(R+t)$ as the set obtained from $R$ by adding $t$ to each element of $R$. Note that if the $R$-graph $H$ is not contained in a blow-up of chain $C^{R}$, then $S^{t}(H)$ is not contained in a blow-up of the chain $C^{(R+t)}$. Thus we have the following fact:

Corollary 19. Let $H$ be a non-trivial degenerate $R$-graph, let $t$ be any positive integer. Then the $t$-times suspension $S^{t}(H)$ is a non-trivial degenerate $(R+t)$-graph.

Lemma 20. Given a positive integer $t \geqslant 2$, and a $\{1, t\}$-graph $H$, let $T(H)$ be the $\{1, t+1\}$-graph obtained from $H$ by adding a new vertex $v \notin V(H)$ such that $V=$ $V(H) \cup\{v\}, T(H)^{1}=H^{1}$ and $T(H)^{1+t}=\left\{e \cup\{v\}: e \in E\left(H^{t}\right)\right\}$. Then we have $\pi(T(H)) \leqslant \pi(H)$.

Proof. Let $n$ be a positive integer and $G=(V, E)$ be an extremal $T(H)$-free $\{1, t+1\}$ graph on $n$ vertices. We have $\pi_{n}(T(H))=h_{n}(G)$. Denote $E_{i}$ as the set of $i$-edges of $G$, for $i=1, t+1$. For any vertex $v \in V(G)$, denote $G_{v}$ as the hypergraph obtained from $G$ with the vertex set $V\left(G_{v}\right)=V \backslash\{v\}$ and the edge sets $E\left(G_{v}\right)=E_{v, 1} \cup E_{v, t}$, where $E_{v, 1}=\left\{u \in V\left(G_{v}\right): u \in E_{1}\right\}$ and $E_{v, t}=\left\{\left\{u_{1}, \ldots, u_{t}\right\}:\left\{v, u_{1}, \ldots, u_{t}\right\} \in E_{t+1}\right\}$. Observe that $G_{v}$ is an $H$-free $\{1, t\}$-graph on $n-1$ vertices. Thus $h_{n-1}\left(G_{v}\right) \leqslant \pi_{n-1}(H)$.

Since

$$
\left|E_{1}\right|=\frac{1}{n-1} \sum_{v \in V(G)}\left|E_{v, 1}\right| \quad \text { and } \quad\left|E_{t+1}\right|=\frac{1}{(t+1)} \sum_{v \in V(G)}\left|E_{v, t}\right|
$$


then

$$
\begin{aligned}
h_{n}(G) & =\frac{\left|E_{1}\right|}{\left(\begin{array}{c}
n \\
1
\end{array}\right)}+\frac{\left|E_{1+t}\right|}{\left(\begin{array}{c}
n \\
1+t
\end{array}\right)} \\
& =\sum_{v \in V(G)} \frac{\left|E_{v, 1}\right|}{(n-1)\left(\begin{array}{c}
n \\
1
\end{array}\right)}+\sum_{v \in V(G)} \frac{\left|E_{v, t}\right|}{(t+1)\left(\begin{array}{c}
n \\
1+t
\end{array}\right)} \\
& =\frac{1}{n} \sum_{v \in V(G)}\left(\frac{\left|E_{v, 1}\right|}{\left(\begin{array}{c}
n-1 \\
1
\end{array}\right)}+\frac{\left|E_{v, t}\right|}{\left(\begin{array}{c}
n-1 \\
t
\end{array}\right)}\right) \\
& =\frac{1}{n} \sum_{v \in V(G)} h_{n-1}\left(G_{v}\right) \\
& \leqslant \pi_{n-1}(H) .
\end{aligned}
$$

Thus $\pi(T(H))=\lim _{n \rightarrow \infty} \pi_{n}(T(H))=\lim _{n \rightarrow \infty} h_{n}(G) \leqslant \pi(H)$.

Lemma 21. Let $R$ be a set of two distinct positive integers, $R \neq\{1,2\}$. Then there exist non-trivial degenerate $R$-graphs.

Proof. By Corollary 19, for every positive integer $k$, one can take the suspension of $H_{5}^{\{1,3\}}$ $k$-times, the resulting graph $S^{k}\left(H_{5}^{\{1,3\}}\right)$ is a non-trivial degenerate $\{1+k, 3+k\}$-graph. Thus there are non-trivial degenerate hypergraphs of edge types: $\{1,3\},\{2,4\},\{3,5\}$, $\ldots,\{k, k+2\}, \ldots$

In [4], the authors found a non-trivial degenerate $\{2,3\}$-graph: $H_{4}^{\{2,3\}}=\{12,13,234\}$. Similarly, by Corollary 19, there are non-trivial degenerate hypergraphs of edge types: $\{2,3\},\{3,4\},\{4,5\}, \ldots,\{k, k+1\}, \ldots$

Using Lemma 20 on $H_{5}^{\{1,3\}}$, there are non-trivial degenerate hypergraphs of edge types: $\{1,4\},\{1,5\}, \ldots,\{1, t\}, \ldots$, for integer $t \geqslant 4$. For each of these non-trivial degenerate $\{1, t\}$-graphs, applying Corollary 19, there are non-trivial degenerate hypergraphs of edge types: $\{2,1+t\},\{3,2+t\}, \ldots,\{k, k-1+t\}, \ldots$.

To summarize, for each integer $k \geqslant 2$ and each integer $t \geqslant 3$, we have non-trivial degenerate hypergraphs of edge types $\{1, t\},\{k, k+1\},\{k, k+2\},\{k, k+t\}$, which cover all sets of two distinct positive integers, except $\{1,2\}$.

Lemma 22. Let $R$ be a set of distinct positive integers with $|R| \geqslant 2$ and $1 \notin R$. If there exist non-trivial degenerate $R$-graphs, then there exist non-trivial degenerate $\{1\} \cup R$ graphs.

Proof. For each $R$ stated in the lemma, let $H$ be the non-trivial degenerate $R$-graph. Let $H^{\prime}$ be the disjoint union of $H$ with a single 1-edge $v \notin H$. Clearly, $H^{\prime}$ is not contained in a blow-up of chain $C^{\{1\} \cup R}$. We will prove that $H^{\prime}$ is also degenerate.

Let $n$ be a positive integer and $G=(V, E)$ be an extremal $H^{\prime}$-free $\{1\} \cup R$-graph on $n$ vertices. We have $\pi_{n}\left(H^{\prime}\right)=h_{n}(G)$. Denote $E_{i}$ as the set of $i$-edges of $G$, for each $i \in\{1\} \cup R$. For any 1-edge $v \in E_{1}$, consider the sub-graph $G_{v}$ of $G$ by removing all 
1-edges (keep the vertices of these 1-edges in $G_{v}$ ). Then the vertex set $V\left(G_{v}\right)=V$, set of $i$-edges $E_{i}\left(G_{v}\right)=E_{i}(G)$ for each $i \in R$. Then we have

$$
\left|E_{i}(G)\right|=\frac{1}{\left|E_{1}\right|} \sum_{v \in E_{1}}\left|E_{i}\left(G_{v}\right)\right|, \forall i \in R .
$$

Observe that $G_{v}$ is an $H$-free $R$-graph on $n$ vertices, so $\pi_{n}(H) \geqslant h_{n}\left(G_{v}\right)$. Then we have

$$
\begin{aligned}
h_{n}(G) & =\sum_{i \in\{1\} \cup R} \frac{\left|E_{i}\right|}{\left(\begin{array}{c}
n \\
i
\end{array}\right)} \\
& =\frac{\left|E_{1}\right|}{\left(\begin{array}{c}
n \\
1
\end{array}\right)}+\sum_{i \in R} \sum_{v \in E_{1}} \frac{\left|E_{i}\left(G_{v}\right)\right|}{\left|E_{1}\right|\left(\begin{array}{c}
n \\
i
\end{array}\right)} \\
& \leqslant 1+\frac{1}{\left|E_{1}\right|} \sum_{v \in E_{1}} h_{n}\left(G_{v}\right) \\
& \leqslant 1+\pi_{n}(H) .
\end{aligned}
$$

Thus $\pi\left(H^{\prime}\right)=\lim _{n \rightarrow \infty} \pi_{n}\left(H^{\prime}\right)=\lim _{n \rightarrow \infty} h_{n}(G) \leqslant 1+\pi(H)=|R|$, then $\pi\left(H^{\prime}\right)=|R|$. Therefore, $H^{\prime}$ is a non-trivial degenerate $\{1\} \cup R$-graph.

Proof of Theorem 3. Using the non-trivial degenerate $R$-graph for $R$ stated in Lemma 21, then apply Lemma 22, we obtain non-trivial degenerate $R$-graphs for $|R|=3$ and $1 \in R$. Apply Corollary 19, we then obtain all other non-trivial degenerate $R$-graphs for $|R|=3$. Repeatedly apply Lemma 22 and Corollary 19, we can obtain all $R$-graphs for $|R| \geqslant 4$, the result follows.

We conjecture that for any set $R$, there exists an $R$-graph $H^{R}$ such that if $G^{R}$ is $R$ degenerate if and only if $G^{R}$ is $H^{R}$-colorable. This conjecture is true for the case $R=\{r\}$ with $r \geqslant 2$ and $R=\{1,2\}$ and is confirmed for $R=\{1,3\}$ in this paper.

\section{References}

[1] P. Erdös and M. Simonovits. A limit theorem in graph theory, Studia Sci. Math. Hungar., 1 (1966), 51-57.

[2] P. Erdös and A. H. Stone. On the structure of linear graphs, Bull. Amer. Math. Soc. 52 (1946), 1087-1091.

[3] P. Erdős, On some extremal problems in graph theory, Israel J. Math., 3 (1965), 113-116.

[4] T. Johnston and L. Lu. Turán problems on non-uniform hypergraphs, Electronic Journal of Combinatorics, 21(4) \#P4.22 (2014).

[5] T. Johnston and L. Lu. Strong jumps and Lagrangians of non-uniform hypergraphs, available at arXiv:1403.1220. 
[6] G. Katona, T. Nemetz and M. Simonovits. On a problem of Turán in the theory of graphs, Mat. Lapok 15 (1964), 228-238.

[7] D. Mubayi and Y. Zhao. Non-uniform Turán-type problems, J. Comb. Th. A, 111 (2004), 106-110.

[8] Y. Peng, H. Peng, Q. Tang and C. Zhao. An extension of the Motzkin-Straus theorem to non-uniform hypergraphs and its applications, Discrete Applied Mathematics, 200 (2016), 170-175.

[9] Yuejian Peng, Biao Wu, Yuping Yao. A Note on Generalized Lagrangians of Nonuniform Hypergraphs, Order 34 (2017), 9-21.

[10] P. Turán. On an extremal problem in graph theory, Mat. Fiz. Lapok 48 (1941), 436-452. 